\title{
Mediastinal Follicular Lymphoma Diagnosed with Multidirectional Analysis Using Tissue Samples Obtained by EBUS-TBNA
}

\author{
Masahiro Inoue ${ }^{1}$, Takahiro Nakajima ${ }^{1}$, Hideki Tsujimura ${ }^{2}$, Makiko Itami ${ }^{3}$, Yuichi Sakairi ${ }^{1}$, \\ Hideki Kimura $^{1}$ and Toshihiko Iizasa ${ }^{1}$
}

\begin{abstract}
Endobronchial ultrasound-guided transbronchial needle aspiration (EBUS-TBNA) has been developed as a useful tool for the diagnosis of diseases adjacent to the airway to be used in place of open surgery or mediastinoscopy. In this report, we present a 46-year-old man with a solid tumor in the mediastinum. Since no other pathological sites were identified, EBUS-TBNA was employed for tumor sampling. Sufficient tissue specimens were collected and the diagnosis of follicular lymphoma was established based on the combination of immunohistochemistry, flow cytometry and FISH analysis. This report demonstrates the advantage of EBUS-TBNA even for the diagnosis of hematological disease.
\end{abstract}

Key words: follicular lymphoma, endobronchial ultrasound-guided transbronchial needle aspiration, flow cytometry, fluorescence in-situ hybridization

(Inter Med 49: 2147-2149, 2010)

(DOI: 10.2169/internalmedicine.49.3213)

\section{Introduction}

The treatment strategy of malignant lymphoma is determined based on the histological diagnosis. For an accurate diagnosis, sufficient amounts of tumor tissues are required and open biopsy of disease sites has been recommended even when the tumor is within the thoracic and abdominal cavities. However, open surgery sometimes induces severe problems including bleeding, infection or organ injuries.

Recently, we have developed an endobronchial ultrasound-guided transbronchial needle aspiration (EBUSTBNA) as a useful tool for the diagnosis of diseases within or adjacent to the airway in particular for use in the field of lung cancer (1-3). In this report, we describe a patient with follicular lymphoma which was localized in the mediastinum. We obtained tumor tissue by using EBUS-TBNA and successfully established the diagnosis. Here we show the advantage of EBUS-TBNA to provide a valuable insight into the clinical management of hematological neoplasm.

\section{Case Report}

A 46-year-old man was referred to our hospital because of an abnormality on chest X-ray examination performed as an annual medical check. The CT scan showed a mediastinal tumor $(75 \times 40 \times 40 \mathrm{~mm})$ which involved the trachea and main bronchus (Fig. 1A). Imaging studies for whole body did not show any other pathological sites except for small lymphadonopathy up to $10 \mathrm{~mm}$ in the abdomen. The patient did not show any symptoms at this time. In addition, no abnormal findings were seen in laboratory examinations including complete blood count, biochemistry, tumor markers and soluble IL2 receptor. To obtain the tumor specimen for the histological diagnosis, EBUS-TBNA by using a 22gauge TBNA needle was performed as previously described (1) (Fig. 1B). Adequate specimens were obtained by 4 punctures from the right upper paratracheal lymph node $(\# 2 R)$ and histological study and fluorescence in-situ hybridization (FISH) analysis on paraffin embedded tissue

${ }^{1}$ Division of Thoracic Diseases, Chiba Cancer Center, Chiba, ${ }^{2}$ Division of Clinical Pathology, Chiba Cancer Center, Chiba and ${ }^{3}$ Division of Hematology-Oncology, Chiba Cancer Center, Chiba

Received for publication December 2, 2009; Accepted for publication July 6, 2010

Correspondence to Dr. Hideki Tsujimura, htsujimu@chiba-cc.jp 

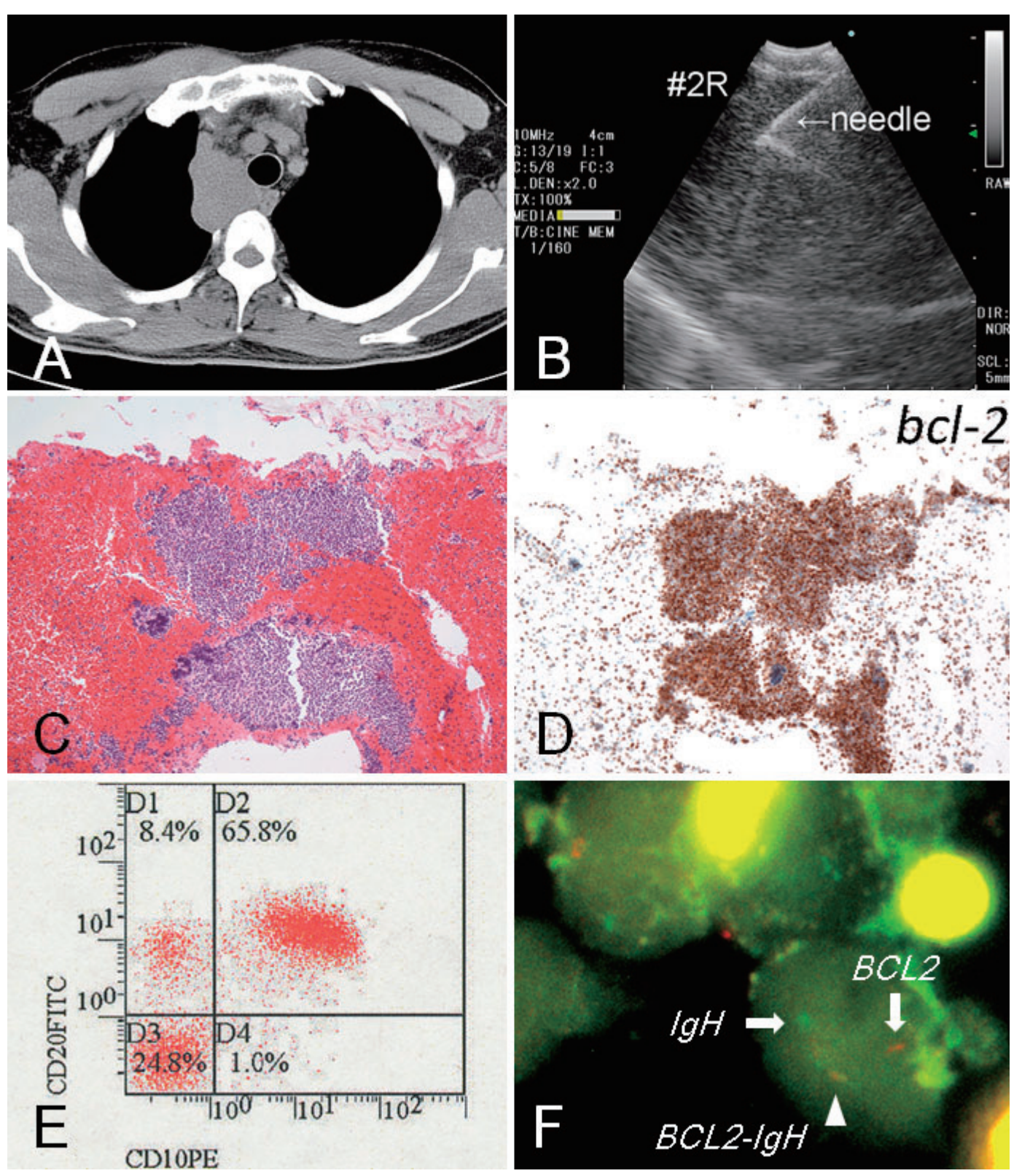

Figure 1. A: Chest CT shows an enlarged paratracheal lymph node (\#2R). B: EBUS-TBNA was performed on theparatracheal lymph node $(\# 2 R)$. C: Tissue sample obtained by EBUS-TBNA shows monotonous proliferation with small and middle sized atypical lymphocytes. D: Malignant cells are positive for bcl-2 by immunohistochemistry. E: Flow cytometry shows that suspended cells collected from TBNA needle are double positive for CD10 and 20. F: FISH analysis demonstrates fusion of $B C L 2-I g H$ (arrowhead) in 59\% of analyzed cells. Arrows indicate BCL2 (red) and IgH (green).

were performed. For FISH analysis, Vysis FISH DNA probe detecting the $B C L 2-I g H$ fusion gene (Illinois, USA) was applied according to the manufacture instruction. In addition, remained cells in the aspiration needles were collected by washing with saline and referred for flow cytometry. Histological study showed monotonous proliferation with small and middle sized atypical lymphocytes (Fig. 1C). Although the samples were not of sufficient size to provide the evidence of follicular formation, immunohistochemistry demonstrated that the tumor cells expressed CD10, CD20 and BCL-2 suggesting B-cell lymphoma originated from follicu- lar center (Fig. 1D). Flow cytometric analysis also showed that tumor cells were positive for CD10, 19, 20 and negative for $\mathrm{CD} 2,3,5,7$ and 56 (Fig. 1E). Finally, FISH analysis demonstrated fusion of BCL2-IgH in 59\% of analyzed cells indicating that $\mathrm{t}(14 ; 18)(\mathrm{q} 32 ; \mathrm{q} 21)$ was involved in malignant cells (Fig. 1F). With these findings, the diagnosis of follicular lymphoma was established. After the diagnosis, the patient went to another hospital for chemotherapy. During the diagnosis process, no documented complication such as bleeding, infection and organ injury was observed as previously reviewed (2). 


\section{Discussion}

With the recent advances in technology, real-time EBUSguided TBNA has been developed and several publications have documented high diagnostic values on pathological sites adjacent to the central airway (1-3). Although the main indication for EBUS-TBNA is lymph node staging of lung cancer, it can also be used for the investigation of unknown mediastinal lymphadenopathy and tumors (2). Importantly, the tissue samples obtained by this technique are also feasible for genetic analysis represented by the epidermal growth factor receptor (EGFR) mutation assay $(4,5)$.

Since the mediastinum is one of the major sites of lymphoma development, some investigators applied EBUSTBNA for diagnosis $(6,7)$. Kennedy et al retrospectively reviewed 236 patients with mediastinal lymphadenopathy referred for EBUS-TBNA in their institutes (7). In their report, they presented 25 patients in whom lymphoma was suspected based on prior history or clinical presentation. They obtained adequate lymph node tissue samples in 24 patients and identified lymphoma in 10 patients and benign disease in 14 patients. The sensitivity, specificity, positive predictive value and negative predictive value observed were $90.0 \%, 100 \%, 100 \%$ and $92.6 \%$, respectively. Although their work suggested the potential of EBUS-TBNA in the diagnosis process of lymphoma, the role of EBUS-TBNA has not yet been well defined because of the limitation of sample yield.

Currently, the diagnosis of lymphoma is based on the World Health Organization (WHO) classification (8). This classification endorses a multiparametric approach to diagnosis with identification of morphologic, phenotypic and genotypic features that are characteristic of each disease entity. For the morphological analysis, sufficient amounts of tumor specimen are still required. However, a relatively small sized sample enables phenotypic or genetic analysis such as flow cytometry, FISH and PCR analysis to be completed.

The present case showed an unknown mediastinal tumor. Options for tissue diagnosis include open surgery, mediastinoscopy or EBUS-TBNA. Since the disease showed indolent presentation and immediate treatment was not necessary, we first employed EBUS-TBNA which can be done on an outpatient basis. On the other hand, if a patient's condition is serious with disease progression or any complications other than respiratory failure, this procedure could also be considered because of its safety.

Although the morphological diagnosis of the current patient was not fixed because of the limitations of tissue yield, the combination of immunohistochemistry, flow cytometry and FISH analysis confirmed the diagnosis of follicular lym- phoma. In the case of lymphomas showing distinct phenotype or genetic abnormality such as the current case, these examinations may support the accurate diagnosis even when the tissue size is limited. However, localized mediastinal lymphoma often presents precursor $\mathrm{T}$ lymphoblastic lymphoma, primary mediastinal large B-cell lymphoma or Hodgkin lymphoma. These subtypes need distinct treatment strategies and the highest priority should be given to the diagnostic accuracy. To determine if EBUS-TBNA is available for the diagnosis of these entities instead of more invasive methods such as mediastinoscopy, further investigations are required.

\section{Acknowledgement}

We would like to thank Dr. Kazuhiro Yasufuku for his helpful suggestions of this manuscript. We also wish to thank Dr. Saori Kirishi and Dr. Hideki Katsura (TWMU Yachiyo Medical Center) for the clinical information.

All authors read the final manuscript. All authors have no conflict of interest related with this article.

Financial support: This research was supported, in part, by the Ministry of Education, Culture, Sports, Science and Technology, Grant-in-Aid for Young Scientists (B) No. 21791340 in 2009 (TN).

\section{References}

1. Yasufuku K, Nakajima T, Motoori K, et al. Comparison of endobronchial ultrasound, positron emission tomography, and CT for lymph node staging of lung cancer. Chest 130: 710-718, 2006.

2. Yasufuku K, Nakajima T, Fujiwara T, et al. Role of endobronchial ultrasound-guided transbronchial needle aspiration in the management of lung cancer. Gen Thorac Cardiovasc Surg 56: 268-276, 2008.

3. Nakajima T, Shingyouji M, Nishimura H, et al. New endobronchial ultrasound imaging for differentiating metastatic site within a mediastinal lymph node. J Thorac Oncol 4: 1289-1290, 2009.

4. Nakajima T, Yasufuku K, Suzuki M, et al. Assessment of epidermal growth factor receptor mutation by endobronchial ultrasoundguided transbronchial needle aspiration. Chest 132: 597-602, 2007.

5. Nishimura H, Nakajima $T$, Itakura $M$, et al. Successful treatment of lung cancer with gefitinib and EGFR mutation status determination using EBUS-TBNA samples in an extremely old patient. Intern Med 48: 1905-1907, 2009.

6. Bangerter M, Brudler O, Heinrich B, Griesshamnuer M. Fine needle aspiration cytology and flow cytometry in the diagnosis and subclassification of non-Hodgkin's lymphoma based on the World Health Organization classification. Acta Cytol 51: 390-398, 2007.

7. Kennedy MP, Jimenez CA, Bruzzi JF, et al. Endobronchial ultrasound-guided transbronchial needle aspiration in the diagnosis of lymphoma. Thorax 63: 360-365, 2008.

8. Swerdlow SH, Campo E, Harris NL, et al. WHO Classification of Tumours of Haematopoietic and Lymphoid Tissues. IARC Press, Lyon, France, 2008.

(C) 2010 The Japanese Society of Internal Medicine http://www.naika.or.jp/imindex.html 